\title{
COMPRESIONES RADICULARES EN LA COLUMNA LUMBAR
}

\author{
Dr. HUMBERTO RAMOS \\ Clínica del Seguro, Quito
}

En las estadísticas de admisión a los servicios asistenciales del Seguro Social en Quito, el problema del dolor lumbar bajo (lumbociatalgia), presenta un elevado porcentaje. En la etiopatogenia de este síndrome se reconoce como causa final la compresión radicular; pero, en general, existen una serie de factores predisponentes y determinantes que son los que llevan en forma progresiva al establecimiento de este cuadro clínico.

Entre los factores predisponentes, el más importante es, sin duda alguna, el desconocimiento de normas elementales, en cuanto se refiere a la adopciór de una normal posición erecta o sentada del individuo para la ejecución de las diversas tareas domésticas o de trabajo ${ }^{1}$. Por otro lado, es importante considerar que gran parte de estos pacientes son obreros que, como parte de sus actividades diarias, deben realizar considerables esfuerzos físicos que afectan a la integridad del eje vertebral óseo-muscular.

En cuanto a los factores determinantes de las lumbociatalgias, los más frecuentes son los siguientes:

Factores óseos-.Fracturas de pedículo, de platillo vertebral, luxofracturas vertebrales, espondilolistesis (con arco neural posterior flotante o fracturado), hipertrofia ósea del reborde de los platillos vertebrales, artrosis interapofisarias, compresión postdesis de columna vertebral.

Factores por lesión de la raíz misma.-Várices de raíz, edema, hematoma, adherencias de la raíz a su vaina, aracnoiditis, epiduritis. Tumoraciones extra o intradurales. Hipertrofia del ligamento amarillo. Hernias discales.

Diagnóstico.-La identificación o diagnóstico de la lesión ósea determinante del cuadro clínico, a menudo ofrece serias dificultades. Sin embargo, una cuidadosa historia clínica complementada con un prolijo examen físico, permiten una presunción diagnóstica que puede confirmarse o rectificarse con el auxilio de los exámenes complementarios de laboratorio, especialmente del correspondiente estudio radiológico $^{2-3}$.

Cuadro Clínico.-Tiene especial in- 
terés la investigación de antecedentes, causas mediatas o inmediatas para la aparición del dolor, naturaleza de éste, irradiación, relación con esfuerzos, decúbitos y con la marcha. En el examen físico debe ponerse mucho cuidado en la búsqueda de signos de elongación del ciático, disminución o aumento del dolor con los diferentes decúbitos, con la marcha, tonicidad muscular, reflejos osteo-tendinosos, cutáneos y sensibilidad en sus diferentes formas ${ }^{4}$.

Exámenes Auxiliares.-Aparte de los exámenes de laboratorio, principalmente el hematológico y las pruebas de electrodiagnóstcio, uno de los más importantes es el radiológico. En muchas ocasiones una radiografía simpie, en posiciones adecuadas según el casc. permite una solución diagnóstica. En otros casos hay que recurrir a técnicas especiales ${ }^{3}$, tales como la neumografía y la mielografía, que tienen el grave inconveniente de producir lesiones o secuelas si no graves, por lo menos muy molestosas para el paciente.

Cuando se tiene el diagnóstico provisional de hernia discal posterior y media, la neumografía es una indicación precisa; esta técnica tiene la ventaja de ser simple y, por lo tanto, fácilmente ejecutable; presenta además apreciable seguridad diagnóstica. La lectura de la placa radiográfica es fácil en los segmentos altos de la columna, no así entre el espacio intervertebral VL. y I sacro, donde la sombra del ilíaco dificulta la visibilidad. Este inconveniente se ha obviado tomando tomografías en diferentes planos de esta área anatomotopográfica.

La mielografía con contraste positivo, practicada en forma rutinaria por varios autores y objetada por otros, tiene su indicación precisa en los casos de tumoraciones intra o extradurales, compresiones radiculares post-operatorias y en casos cuya solución diagnóstica no ha sido posible, por otros medios, incluyendo la neumomielografía. La mielografía con contraste positivo tiene la ventaja de permitir una excelente visualización del espacio vertebral. En cambio, presenta la desventaja de provocar procesos irritativos de meninges, con secuelas que se traducen por síndromes dolorosos tanto o más intensos que los iniciales, objeto de la investigación diagnóstica. Esta complicación no se presenta inmediatamente sino transcurrido algún tiempo, inclusive años, después de realizado el examen.

Este inconveniente ha tratado de sólucionarse con la extracción del medio de contraste, luego de terminado el estudio radiológico y con la ayuda de la fluoroscopia; lamentablemente, este procedimiento, no en todos los casos ha dado buen resultado. Por lo tanto, en los momentos actuales, se tiende más bien a utilizar substancias yodadas no oleosas que se reabsorben totalmente y que, según investigaciones preliminares, parece que no se presentan tales efectos colaterales.

En el servicio de Traumatología y Ortopedia de la Clínica del Seguro Social en Quito, de 134 pacientes adm:tidos con el diagnóstico de dolor ?umbar bajo, se han realizado, en total, 64 neumomielografías y 15 mielografías con contraste positivo. La positividad diagnóstica fue, aproximadamente, del 
$90 \%$, comprobada con la respectiva intervención quirúrgica.

En aquellos pacientes en que se realizó mielografía en contraste positivo, se observó que después de días, e inclusive de algunos meses, se presentaron neuritis y radiculitis con síndromes dolorosos intensos. En algunos de estos pacientes, controlados luego de varios años, la substancia de contraste aún estuvo presente en los fondos de saco radiculares, visualizándose a manera de grumos pequeños que siguen el trayecto de las raíces hasta el sitio donde emergen del canal radicular. En uno de los casos inclusive se intentó drenar quirúrgicamente estos residuos, sin resultados satisfactorios.

Las intervenciones quirúrgicas realizadas en los años 62 y 63 se efectuaban con una laminectomía media y poco lateralizada; en los años que siguen la laminectomía se la hace más lateralizada llegando incluso a las carillas interapofisarias y agrandando el agujero conjuntival.

Hallazgos operatorios.-En la tabla I se resumen los datos relacionados con el diagnóstico clínico presuntivo de 134 pacientes. De éstos, solamente 65 fueron sometidos a la intervención quirúrgica respectiva; en la mayoría de ellos se ratificó el diagnóstico clínico; en otros hubo rectificación o constituyeron hallazgos operatorios.

En los casos de espondilolistesis, el arco neural posterior se encontró totalmente móvil y en dos casos hubo fractura de lámina. Se realizó la laminectomía descomprensiva y desis de columna, empleando auto, hétero injerto óseo, o ambos a la vez.
En el caso de cabalgamiento de láminas vertebrales con compresión radicular, (el cabalgamiento fue tan pronunciado que semejaba una artrodesis), al efectuar la laminectomía en la zona externa cercana a la articulación interapofisaria resecando con la pinza de Dandy se extirpó accidentalmente un filete nervioso delgado, de una longitud de 2,5 a $3 \mathrm{~cm}$., se trataba del nervio de Luchka. Sin embargo, el paciente se recuperó sin presentar problema posterior alguno.

En los casos de compresión por injerto óseo en desis de columna se ha observado un crecimiento exuberante del injerto óseo hacia porción anterior llegando a presionar sobre raíces y saco dural. En uno de estos casos la compresión fue tan acentuada que la imagen mielográfica se la podía comparar con un reloj de arena. Actualmente, tratamos de obviar este inconveniente colocando el injerto óseo en el sitio de la laminectomía mirando la porción cortical a la ventana del canal vertebral, con el objeto de que la proliferación ósea se realice en tejido esponjoso o sea en la parte posterior del injerto.

La hipertrofia del ligamento amarillo va en algunos casos hasta un centímetro de espesor dando la compresión radicular; en casos en los cuales existe un signo de Dandy positivo, esta hipertrofia se explica fácilmente.

Tratándose de hernias discales se han encontrado núcleos pulposos casi en su totalidad protruídos y otras protrucciones discales (hernias ocultas) que no llegan a romper el ligamento vertebral común posterior. En mayor porcentaje se encuentran hernias entre 
Tabla I

DIGNOSTICO PRESUNTIVO Y DIAGNOSTICO POST-QUIRURGICO DE 143 CASOS DE COMPRESIONES RADICULARES

CLINICA DEL SEGURO SOCIAL

Quito, 1962 - 1964

\begin{tabular}{|c|c|c|c|c|}
\hline \multirow[t]{2}{*}{ Tipo de lesión } & \multicolumn{2}{|c|}{$\begin{array}{l}\text { Diágnóstico } \\
\text { Presuntivo }\end{array}$} & \multicolumn{2}{|c|}{$\begin{array}{c}\text { Diágnóstico } \\
\text { post-quirúrgico }\end{array}$} \\
\hline & No & $\%$ & $\mathrm{~N}^{0}$ & $\%$ \\
\hline $\begin{array}{l}\text { Compresiones radiculares } \\
\text { (sin especificación) } \ldots \ldots \ldots \ldots\end{array}$ & 39 & 28 & - & \\
\hline Hernias discales $\ldots \ldots \ldots \ldots$ & 31 & 22 & 20 & 31,5 \\
\hline Espondilolistesis $\ldots \ldots \ldots \ldots$ & 30 & 20 & 8 & 12,5 \\
\hline Compresión por injerto óseo ... & 3 & 2 & 3 & 4,5 \\
\hline Várices de raíz $\ldots \ldots \ldots \ldots \ldots$ & 5 & 3,5 & 5 & 7,5 \\
\hline Fracturas (luxofracturas) $\ldots$. & 14 & 9,5 & 11 & 17,5 \\
\hline $\begin{array}{l}\text { Hipertrofia del ligamento ama- } \\
\text { rillo } \ldots \ldots \\
\ldots\end{array}$ & 10 & 8 & 10 & 15 \\
\hline $\begin{array}{l}\text { Espícula ósea de platillo ver- } \\
\text { tebral } \ldots \ldots \ldots \ldots \ldots \ldots \ldots \ldots\end{array}$ & 2 & 1,5 & - & \\
\hline Radiculitis post-mielográfica $\ldots$ & 2 & 1,5 & - & \\
\hline Fibroma del canal vertebral .... & 1 & 0,5 & 1 & 1,5 \\
\hline Paquimeningitis $\ldots \ldots c c c c c$ & 4 & 2,5 & 4 & 6,5 \\
\hline $\begin{array}{l}\text { Cabalgamiento de láminas verte- } \\
\text { brales } \ldots \ldots \ldots \ldots \ldots \ldots \ldots\end{array}$ & 1 & 0,5 & 1 & 1,5 \\
\hline $\begin{array}{l}\text { Fractura injerto óseo de desis de } \\
\text { columna } \ldots \ldots \ldots \ldots \ldots\end{array}$ & 1 & 0,5 & 1 & 1,5 \\
\hline Osteofitos marginales $\ldots \ldots \ldots$ & - & & 1 & 1,5 \\
\hline Total $\ldots \ldots \ldots \ldots$ & 143 & 100 & 65 & 90 \\
\hline
\end{tabular}


las vértebras quinta lumbar y primera sacra, siguiendo las de cuarto espario intervertebral y pocas en tercer espacio.

La evaluación de la capacidad física post-operatoria del individuo ha determinado un 90 a $100 \%$ de recuperación, tratándose de extirpación de hernias discales. Son pacientes que pueden retornar a su trabajo y aún practicar deportes que exigen esfuerzos físicos considerables.

Se observa que si la intervención quirúrgica se la realiza antes que la compresión se manifieste clínicamente por síntomas y signos de degeneración nerviosa (disminución de tonicidad y volumen muscular) la recuperación del paciente es más rápida y compieta.

Las lumbalgias post-radiculares (residuales) que se presentan son de intensidad mínima o mediana y ceden con medicación sintomática.

Las intervenciones en las cuales se ha realizado artrodesis de columna deja incapacidad de un 30 a $40 \%$ para la flexión de la columna. Aquellos casos que necesitaron de una nueva intervención quirúrgica dieron mayor porciento de incapacidad y quedaron con lumbalgias residuales de mediana o gran intensidad.

\section{RESUMEN}

El dolor lumbar bajo (lumbociatalgia) es una de las causas más frecuentes de admisión al Servicio de Traumatología y Ortopedia de la Clínica del Seguro Social, en Quito. Desde 1962 hasta Junio de 1965, se admitieron 134 pacientes con el diagnóstico provisional de lumbociatalgia por compresión radicular; de éstos, solamente 65 fueron intervenidos quirúrgicamente.

En aquellos casos de difícil solución diagnóstica, se recurrió al examen radiológico de columna lumbar. Se efectuaron 64 neumomielografías y 15 mielografías con contraste positivo. La positividad diagnóstica de estos medios auxiliares fue del $90 \%$.

Las lesiones anatómicas más frecuentes, identificadas clínica o quirúrgicamente fueron las siguientes: com. presiones radiculares sin especificación, 39 (28\%); hernias discales, 31 (22\%), espondilolistesis, $30(20 \%)$; luxo- fracturas, 14 (9,5\%); hipertrofia del ligamento amarillo, 10 (8\%).

\section{SUMMARY}

The low lumbar pain (lumbosciat:s) is on of the most frequent causes of admission to the Traumatology and Orthopedia Department of the "Clin:ca del Seguro Social" in Quito.

Since 1962 until June, 1965, 134 patients were admitted with the provisional diagnosis of lumbosciatic by radicular compression; of those only 65 were surgically treated.

In cases of difficult diagnosis radiologic inspection of the lumbar spine was done. Sivty four pneumomielographies and 15 mielographies with positive conrtast were done. The possibi- 
lity of diagnosis by this resources was of the $90 \%$. The anatomic lesions clinic and surgically identified in the 134 patients were the follawing: radicular compression without specification 39 $(28 \%)$, discal hernia $31(22 \%)$, spondilolistesis $30(20 \%)$, luxofractures 14 $(9,5 \%)$, hipertrophy of the yellow ligament $10(8 \%)$.

\section{BIBLIOGRAFIA}

1.-SCHELEE, T.: Das lumbosakrade Verfallsyndrom. Med. Klin. 58: 1370, 1963.

2.-BLAN, J. N., and LOGUE, V.: Intermitent claudication of the cauda equina. Lancet I: 1081, 1961.

3.-BARD, M.: Examen radiologique du Rachis Douloureux. Rev. Prat. 11: 3510, 1961.

4.-LANCE, P.: Diagnostic des algies rachidiennes chez l'adulte et chez le vieillard Rev. Prat. 11: 3626, 1961.

\section{MUERTE SUBITA DURANTE EL TRATAMIENTO CON DERIVADOS FENOTIAZINICOS}

Se han registrado seis casos de muerte súbita de pacientes que, fuera de sus trastornos psiquiátricos, podían ser considerados como saludables. La muerte se produjo inesperadamente, existiendo como único antecedente la administración de derivados fenotiazínicos en las dosis usualmente altas que se administra a los pacientes psiquiátricos. En cada caso, el examen patológico post-morten, no dio resultados suficientemente claros como para explicar la muerte. Clínicamente, el ataque comenzó con un episodio sincopal acompañado de convulsiones y seguido rápidamente por la cesación de la respiración y paro cardíaco. En un paciente fue posible una momentánea resucitación, que permitió registrar la fibrilación ventricular, de tipo irreversible.

Al estudiar retrospectivamente la experiencia hospitalaria de muerte de pacientes psiquiátricos, este tipo de muerte apareció definitivamente asociada con la administración de derivados fenotiazínicos. El mecanismo dominante, es probablemente, la fibrilación ventricular aunque también hay que considerar la posibilidad de asfixia durante el ataque convulsivo inducido por las drogas o por la aspiración de alimentos. La naturaleza de lo strastornos cardíacos es aún obscura aunque los estudios recientes sobre anormalidades electrocardiográficas y depósito de pigmentos en el miocardio en pacientes con tratamiento fenotiazínico de larga duración, podría estar relacionada con esta grave complicación que puede originar la muerte del paciente.

(HOLLISTER, L. E. y KOSEK, J. C.: Sudden death during treatment with phenothiazine derivatives. J. A. M. A. 192: 1035, 1965). 